\title{
Bioelectrochemical arsenite oxidation in rice rhizosphere in plant-microbial fuel cells
}

\author{
X.Q. Wang, Y.H. Lv, C.P. Liu, F.B. Li \& Y.H. Du \\ Guangdong Key Laboratory of Integrated Agro-environmental Pollution Control and Management, \\ Guangdong Institute of Eco-Environmental Science \& Technology, Guangzhou, P.R. China
}

\begin{abstract}
The potential for arsenite removal using plant-microbial fuel cells (PMFC) was evaluated. In such a system, $\mathrm{O}_{2}$ is excreted by living rice plant roots and $\mathrm{Fe}(\mathrm{II})$ play important roles in initiating Fenton reactions. Fenton reagents, including $\mathrm{H}_{2} \mathrm{O}_{2}$, $\mathrm{Fe}(\mathrm{II})$, were generated in-situ without an electricity supply. The HO. produced in the Fenton reaction were capable of oxidizing arsenite apparently and efficiently. The results might provide a new insight for As(III) oxidation in rice rhizosphere.
\end{abstract}

\section{INTRODUCTION}

Arsenic (As) is commonly presented as trivalent (arsenite) and pentavalent (arsenate) species in aquatic and soil systems, and the former is much more toxic and mobile than the latter. In flooded paddy soils, $\mathrm{Fe}(\mathrm{III})$ (hydr)oxides can be reduced to $\mathrm{Fe}(\mathrm{II})$, and arsenate adsorbed on the minerals can then be released and reduced to arsenite (Mitsunobu et al., 2006). At the same time, $\mathrm{O}_{2}$ released from the surfaces of rice root can directly oxidize $\mathrm{Fe}$ (II) in rhizosphere to $\mathrm{Fe}$ (III) (hydr)oxides (Colmer et al., 2003). Recently, Wang et al. (2014) used a 'bio-electro-Fenton' reaction system successfully to oxidize and remove arsenite at neutral $\mathrm{pH}$. In wet plant rhizosphere, microorganism activities can generate electricity from the biodegradation of plant root exudates, rhizodeposites and other organic matters. Therefore, we hypothesize that, arsenite can be oxidized to arsenate simultaneously by reactive oxygen species such as HO. via Fenton-like reactions (Wang et al., 2014) and finally incorporated onto Fe(III) (hydr)oxides. As a result of As immobilization, the uptake of As by rice plants may be decreased, e.g., As accumulation in rice may be alleviated.

Consequently, a PMFC was constructed to demonstrate the feasibility of our hypothesis. Arsenite removal efficiencies and kinetics in the PMFC were investigated. The arsenite removal mechanisms in the PMFC were also explored. It should be valuable for understanding the biogeochemical processes of paddies under flooding conditions.

\section{METHODS/EXPERIMENTAL}

PMFC configuration was constructed according to a previously published protocol (Wang et al., 2014). Differently, the PMFC in present study enlarged both the anode and cathode chambers to $600 \mathrm{~mL}$. The PMFC experiments were operated in recycling batch mode. During the experiments, a pure culture of
Shewanella putrefaciens SP200 was used as the biocatalyst in the sterilized anode chamber with $20 \mathrm{mM}$ lactate as the electron donor. Three rice seedlings were planted in the cathode electrode. Rice roots were anticipated to expand along the chamber and be in close contact with the carbon felt $/ \gamma-\mathrm{FeOOH}$ composite cathode. The growth medium contained a $100 \mathrm{mM}$ phosphate buffer-based nutrient solution ( $\mathrm{pH}$ 7.0). $1129 \mu \mathrm{g} \mathrm{L}^{-1}$ arsenite concentration was used to study its removal efficiency by the PMFC. During the startup, an external resistance of $1000 \Omega$ was used to connect the anode and cathode, and the MFC was operated at a controlled temperature of $30^{\circ} \mathrm{C}$. The cell voltages were recorded by a 16-channel voltage collection instrument (AD8223, China).

\section{RESULTS AND DISCUSSION}

\subsection{Arsenite removal in the PMFC}

Although arsenite concentrations in the control reactors under open-circuit conditions decreased lower than that in the PMFC, high arsenite removal efficiencies were found at the end of all the treatments (Fig. 1). In the cathode chamber of the PMFC, more than $90 \%$ arsenite was depleted within $20 \mathrm{~h}$. A small amount of arsenate was detected at $1.5 \mathrm{~h}$, which increased firstly and reached a peak value at $24 \mathrm{~h}$ and then decreased over the subsequent time period. No arsenate was found in the two control experiments. The high arsenite removal efficiency in the two control reactors might be attributed to the adsorption by $\gamma$-FeOOH pasted on the carbon felt. Arsenite removal kinetics in the three reactors were also investigated and were found to follow first-order kinetics with high coefficients of determination $\left(\mathrm{R}^{2}>0.92, \mathrm{p}<0.0001\right)$.

The arsenite removal rate in the PMFC was much higher than those in the control experiments (Table 1). These results indicate that the bioelectrochemical process in the PMFC plays an important role in arsenite 


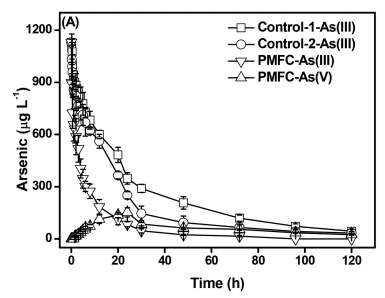

Figure 1. Variation of arsenic concentrations in the PMFC under closed and opened circuit conditions.

Table 1. Performance of PMFC for arsenite removal under various conditions.

\begin{tabular}{lllll}
\hline & $\begin{array}{l}\mathrm{k}_{\mathrm{obs}} \\
\left(\mathrm{h}^{-1}\right)\end{array}$ & $\begin{array}{l}\mathrm{Fe}(\mathrm{II}) \\
\left(\mathrm{mg} \mathrm{L}^{-1}\right)\end{array}$ & $\begin{array}{l}\mathrm{H}_{2} \mathrm{O}_{2} \\
\left(\mathrm{mg} \mathrm{L}^{-1}\right)\end{array}$ & $\begin{array}{l}\mathrm{OCE} \\
(\%)\end{array}$ \\
\hline Control-1 & 0.0271 & $0.57 \pm 0.14$ & & \\
Control-2 & 0.0313 & $0.61 \pm 0.02$ & & \\
PVDF & 0.1107 & $0.88 \pm 0.13$ & $1.58 \pm 0.26$ & 68.4 \\
\hline
\end{tabular}

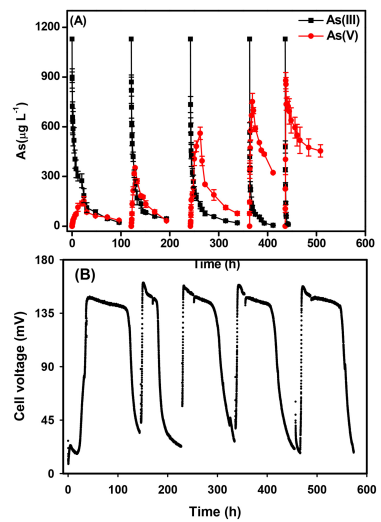

Figure 2. Performances of long-term operation of the PMFC.

oxidation, and the rice plants in the PMFC can stimulate this process. The value of oxidation current efficiency was determined to be $68.41 \%$ in the first $60 \mathrm{~h}$, which was comparable with that of a previous study for Cr(VI) reduction using a PMFC (Habibul et al., 2016).

The repeatable adsorption and oxidation experiments were run for five cycles. Figure 2A shows As species in the aqueous solution of the cathode chamber and the cell voltage of the PMFC over the five cycles. The cycles promoted the depletion rate of arsenite and generation rate of arsenate even if $2 \mathrm{~g}$ of $\gamma-\mathrm{FeOOH}$ was not maintained in the PMFC during the entire experiment. The apparent rate constants from the first to the fifth run were calculated to be $0.11 \pm 0.02$ $\left(\mathrm{R}^{2}=0.95\right), \quad 0.22 \pm 0.02 \quad\left(\mathrm{R}^{2}=0.90\right), \quad 0.50 \pm 0.03$ $\left(\mathrm{R}^{2}=0.91\right), 0.85 \pm 0.13\left(\mathrm{R}^{2}=0.91\right)$ and $0.71 \pm 0.12$ $\left(\mathrm{R}^{2}=0.93\right) \mathrm{h}^{-1}$. The dissolved arsenate increased more and more rapidly from the first cycle to the fifth cycle, implying that the oxidation process plays a more important role than the adsorption process in arsenite depletion. The cell voltage reached a plateau and then started to decrease due to the consumption of lactate in the anode chamber (Fig. 2B) in all the five cycles. These results implied that the root oxygen loss of rice plants prevail for arsenite oxidation in the rhizosphere.

\subsection{Mechanism of arsenite removal in the PMFC and environmental implications}

The increase of arsenate in the cathode chamber indicates that arsenite can be oxidized directly in the cathode coupled with oxidation of organics (lactate) in the anode of the PMFC. The reaction efficiency is highly dependent upon the ROL of living rice plants in the cathode chamber. In addition, the much higher removal rates of arsenite in the PMFC compared with those of the two controls also indicate that the bioelectro-chemical process for arsenite removal is more rapid. As Wang et al. (2014) suggested, this arsenite removal process is important in natural system such as the rhizosphere of rice plants in flooding paddies. In such a system, $\mathrm{O}_{2}$ could be reduced to $\mathrm{H}_{2} \mathrm{O}_{2}$ via accepting electricity generated by microorganism activities, combined with $\mathrm{Fe}$ (II) produced by $\mathrm{Fe}$ (III) (hydr)oxides reductive dissolution, the Fenton reaction was initiated and the resulted $\mathrm{HO}$ - was active for arsenite oxidation.

\section{CONCLUSIONS}

$\mathrm{O}_{2}$ excreted by rice roots can accept electrons derived from microorganism activities and participate in Fenton or Fenton like reactions, which products benefit arsenite oxidation and combination on Fe(III) (hydr)oxides. In summary, the results indicate that bioelectrochemical process plays important roles in arsenite removal in paddy soil-rice systems.

\section{ACKNOWLEDGEMENTS}

This work was financially supported by the National Natural Science Foundation of China (41201504) and Guangdong academy of sciences special funds for the talent with high degree and special technical ability (2017GDASCX-0829).

\section{REFERENCES}

Colmer, T.D. 2003. Long-distance transport of gases in plants: a perspective on internal aeration and radial oxygen loss from roots. Plant Cell. Environ. 26(1): 17-36.

Habibul, N., Yi, H., Wang, Y.K., Wei, C., Yu, H.Q. \& Sheng, G.P. 2016. Bioelectrochemical chromium(VI) removal in plant-microbial fuel cells. Environ. Sci. Technol. 50(7): 3882-3889.

Mitsunobu, S., Harada, T. \& Takahashi, Y. 2006. Comparison of antimony behavior with that of arsenic under various soil redox conditions. Environ. Sci. Technol. 40 (23): 7270-7276.

Wang, X., Liu, C., Yuan, Y. \& Li, F. 2014. Arsenite oxidation and removal driven by a bio-electro-Fenton process under neutral pH conditions. J. Hazard. Mater. 275(2): 200-209. 